\title{
Epigenetic repression of long non-coding RNA MEG3 mediated by DNMT1 represses the p53 pathway in gliomas
}

\author{
JIA LI ${ }^{1,2^{*}}$, ER-BAO BIAN ${ }^{1,2^{*}}$, XIAO-JUN HE ${ }^{1,2}$, CHUN-CHUN MA $^{1,2}$, \\ GANG ZONG $^{1,2}$, HONG-LIANG WANG ${ }^{1,2}$ and BING ZHAO ${ }^{1,2}$ \\ ${ }^{1}$ Department of Neurosurgery, The Second Affiliated Hospital of Anhui Medical University, Hefei, Anhui 230601; \\ ${ }^{2}$ Cerebral Vascular Disease Research Center, Anhui Medical University, Hefei, Anhui 230601, P.R. China
}

Received August 1, 2015; Accepted November 20, 2015

DOI: 10.3892/ijo.2015.3285

\begin{abstract}
Epigenetic regulation plays a significant role in gliomas. However, how methylation and long non-coding RNA (lncRNA) cooperates to regulate gliomas progression is largely unknown. In this investigation we showed that the downregulation of MEG3 expression due to hypermethylation of MEG3 was observed in gliomas tissues. Treatment of glioma cells with the DNA methylation inhibitor 5-Aza-2'-deoxycytidine (5-AzadC) decreased aberrant hypermethylation of the MEG3 promoter and prevented the loss of MEG3 expression. In addition, DNMT1 was involved in MEG3 promoter methylation, and was inversely correlated with MEG3 expression in gliomas. The inhibition of DNMT1 repressed the proliferation, clone formation, and induced apoptosis in glioma cells. Importantly, the inhibition of DNMT1 contributed to the activation of p53 pathways in gliomas cells. These results suggest that DNMT1-mediated MEG3 hypermethylation caused the loss of MEG3 expression, followed by the inhibition of the p53 pathways in gliomas.
\end{abstract}

\section{Introduction}

Malignant gliomas are the most common primary brain tumor in the central nervous system. Gliomas are classified by the 2007

Correspondence to: Professor Bing Zhao, Department of Neurosurgery, The Second Affiliated Hospital of Anhui Medical University, Cerebral Vascular Disease Research Center, Anhui Medical University, 678 Fu Rong Road, Hefei, Anhui 230601, P.R. China

E-mail: aydzhb@126.com

*Contributed equally

Abbreviations: lncRNAs, long non-coding RNAs; MEG3, maternally expressed gene 3; DNMT1, DNA methyltransferase 1; 5-AzadC, 5-aza2-deoxycytidine; DMEM, Dulbecco's modified Eagle's medium; FBS, fetal bovine serum; SP, streptavidin peroxidase; IHC, immunohistochemical; MSP, methylation-specific PCR; RNAi, RNA interference; SiRNA, small interfering RNA; DNMTs, DNA methyltransferases

Key words: gliomas, DNA methylation, DNA methyltransferase 1, long non-coding RNA, maternally expressed gene 3
World Health Organization into four histopathologic grades based on the degree of malignancy (1). Current therapeutic strategies for gliomas consist of surgical resection, radiotherapy and chemotherapy. However, the prognosis of patients suffering from malignant glioma remains poor $(2,3)$. There is a pivotal need for novel molecular targets and approaches to treat this fatal disease. Over the past several decades, genetic studies have implicated number of protein coding genes in the formation and progression of malignant gliomas; however, the function of non-coding RNAs, especially long non-coding RNAs (lncRNAs), in glioma pathogenesis remains largely unknown.

LncRNAs are a class of non-coding RNA transcripts longer than 200 nucleotides with little or no protein-coding capacity (4). Recently, increasing evidence has revealed that more genomic sequences are transcribed into lncRNAs than protein-coding RNAs (5). In addition, a number of studies have shown that significant numbers of IncRNAs are involved in a variety of biological processes through multiple regulation of mechanisms (6-9). MEG3, identified as a member of lncRNAs, is an imprinted gene with maternal expression which encodes a non-coding RNA (10). Dysregulation of MEG3 has been found in various human tumors including hepatocellular carcinoma, renal cell carcinoma and neuroblastoma $(11,12)$. Moreover, MEG3 expression was lost in the majority of clinically non-functioning human pituitary tumors, and it suppressed cancer cell growth, stimulated p53-mediated transcriptional activation, and selectively activated $p 53$ target genes $(13,14)$.

MEG3 expression is under epigenetic control, and the loss of MEG3 expression due to aberrant $\mathrm{CpG}$ methylation of MEG3 has been observed in ovarian cancer (15). Previous studies suggest that DNA methyltransferase 1 (DNMT1) overexpression may be responsible for the aberrant DNA methylation of tumor-related genes in gliomas (16). In addition, 5-aza-2-deoxycytidine (5-AzadC), a DNMT inhibitor, induced re-expression of MEG3 due to demethylation of MEG3 in hepatocellular cancer (17).

It was hypothesized that epigenetic control of MEG3 by DNA methylation would result in the discovery of novel and specific epigenetic regulators of gliomas. The present study determines that DNA methylation mediates the repression of MEG3 transcription in glioma. In addition, experimental 
evidence suggests that hypermethylation of MEG3 mediated by DNMT1 is a molecular cause for glioma.

\section{Materials and methods}

Materials. Dulbecco's modified Eagle's medium (DMEM) and fetal bovine serum (FBS) were obtained from Hyclone (Logan, UT, USA). Lipofectamine 2000, TRIzol ${ }^{\circledR}$ reagent was purchased from Invitrogen (Carlsbad, CA, USA). M-MLV Reverse Transcriptase was purchased from Promega (Madison, WI, USA; cat. M1705). All other chemicals were obtained from Sigma (St.Louis, MO,USA). The antibodies used were as follows: anti-DNMT1 (1:500 dilution; Santa Cruz Biotechnology, Inc.); anti-MDM2 (1:500 dilution; Boster, China); anti-p53 (1:500 dilution; Boster); anti- $\beta$-actin (1:2,000 dilution; Santa Cruz Biotechnology, Inc.). DNA extraction kit was acquired from Axygen. Streptavidin peroxidase (SP) immunohistochemical kit was acquired from Zhongshan Biotechnology Corp. (Beijing, China).

Patients and tissue samples. Tissue samples from normal brain tissues and human glioma tumors were collected from the Neurosurgery Department of The Second Affiliated Hospital of Anhui Medical University (Hefei, China). Samples were collected and snap-frozen in liquid nitrogen immediately and preserved in $-80^{\circ} \mathrm{C}$ until use, and their histological type was further confirmed using the standard hematoxylin and eosin staining according to the WHO criteria. A total of 71 samples were used for this study including primary-grade pilocytic astrocytomas or grade II astrocytomas (WHO I/II, $\mathrm{n}=23$ ), grade III anaplastic astrocytomas or grade IV glioblastoma multiforme (WHO III/IV, n=36) and normal brain tissues derived from the temporal lobes and saddle area of the patients with arachnoid cyst after surgery $(n=12)$, both glioma patients and controls were similar with respect to sex and age. This study was approved by the Research Ethics Committee of The Second Affiliated Hospital of Anhui Medical University. Informed consent was obtained from all the patients.

Immunohistochemistry. We studied 62 glioma patients who had been surgically treated in Department of Neurosurgery, The Second Affiliated Hospital of Anhui Medical University, Hefei, China. For immunohistochemistry, resected glioma tissues were fixed in $10 \%$ formalin solution and embedded in paraffin. Histological slices of $3 \mathrm{~mm}$ were prepared, then deparaffined in xylene, and dehydrated with ethanol. Endogenous peroxidase was blocked with $0.3 \% \mathrm{H}_{2} \mathrm{O}_{2}$ in methanol for $20 \mathrm{~min}$ at room temperature (RT). Following antigen retrieval, the sections were blocked with 5\% BSA for $20 \mathrm{~min}$ at $\mathrm{RT}$ and then probed with 1:500 rabbit anti-DNMT1 at $4^{\circ} \mathrm{C}$ overnight. After washing, the sections were incubated with biotinylated goat anti-rabbit immunoglobulins at RT for $1 \mathrm{~h}$, and visualized using the peroxidase conjugated streptavidin and diaminobenzidine, followed by counterstaining with Mayer's hematoxylin. Results of the immunohistochemical (IHC) staining were evaluated by a pathologist blinded to all clinical data. The criterion for IHC scoring was that a specimen with $>10 \%$ positive staining tumor cells should be considered positive, and our
IHC data for pharyngeal cancer were classed as positive or negative accordingly.

Cell culture. U251, U87 and A172 human glioma cell lines were obtained from the Chinese Academy of Sciences Cell Bank. Cells were maintained in DMEM supplemented with $10 \%$ heat-inactivated FBS and $100 \mathrm{U} / \mathrm{ml}$ penicillin/streptomycin at $37^{\circ} \mathrm{C}$ in humidified atmosphere of $5 \% \mathrm{CO}_{2}$.

5-aza-2'-deoxycytidine treatment. U251 and U87 glioma cells were seeded overnight in culture dishes, 5-AzadC (SigmaAldrich) was added and was refreshed every 24 until $72 \mathrm{~h}$ treatment finished. The medium containing PBS only was regarded as a control.

Methylation-specific polymerase chain reaction. The methylation status of the MEG3 promoter region was determined by methylation-specific PCR (MSP) using bisulfite-modified DNA. Genomic DNA was extracted using the QIAamp DNA mini kit (Axygen). Two primer sets were used to amplify the promoter region of the MEG3 gene that incorporated a number of $\mathrm{CpG}$ sites, one specific for the methylated sequence (MEG3-M, forward, 5'-TATGAGTTGTAAGCGGTAGAGTTC-3'; reverse, 5'-TACGAACTTAACGAAAAAAAATCAT-3') and the other for the unmethylated sequence (MEG3-U, forward, 5'-GAATATGAGTTGTAAGTGGTAGAGTTT-3'; reverse, 5'-TACAAACTTAACAAAAAAAAATCATACT-3'). The primers used in the present study detect specifically the promoter sequence of the MEG3 gene rather than that of the MEG3 pseudogene. For MSP reaction we used the HotStarTaq Master Mix kit (Qiagen). PCR reactions were carried out in a final $25 \mu$ l volume containing: HotStarTaq Master mix (final concentration 1X PCR buffer, 2.5 U Hot Star Taq DNA polymerase, $200 \mu \mathrm{m}$ of each $\mathrm{dNTP}$ and $\left.1.5 \mathrm{mM} \mathrm{MgCl}_{2}\right), 0.4 \mu \mathrm{M}$ primers and bisulphite-modified DNA ( $30 \mathrm{ng})$. Amplification was performed in a thermocycler with the following conditions: $94^{\circ} \mathrm{C}$ for $2 \mathrm{~min}$, cycled at $94^{\circ} \mathrm{C}$ for $30 \mathrm{sec}, 54^{\circ} \mathrm{C}$ or $50^{\circ} \mathrm{C}$ for $30 \mathrm{sec}$, and $72^{\circ} \mathrm{C}$ for $45 \mathrm{sec}$ ( 36 cycles) followed by extension at $72^{\circ} \mathrm{C}$ for $7 \mathrm{~min}$. MSP experiments were performed at least in duplicate.

RNA interference (RNAi) analysis. RNAi experiments in glioma cells were performed by forward transfection in cultured glioma cells ( $2 \times 10^{5}$ cells per $200 \mathrm{~mm}^{2}$ dish) using Lipofectamine RNAiMax (Invitrogen) according to the manufacturer's protocol. For DNMT1, MDM2 and p53 immunoblotting, glioma cells were cultured in serum-free DMEM for $12 \mathrm{~h}$ and then subjected to reverse transfection with RNAiMax in Opti-MEM. Small interfering RNA (siRNA) oligonucleotides against DNMT1 genes or scrambled sequences were synthesized by the Shanghai GenaPharma Corp. The following siRNA sequences were used: si-DNMT1 (human), 5'-GGGACUGUGUCUCUGUUAUTT-3' (sense) and 5'-AUAACAGAGACACAGUCCCTT-3' (antisense); si-control with scrambled sequence (negative control siRNA having no perfect matches to known human genes), 5'-UUCUC CGAACGUGUCACGUTT-3' (sense) and 5'-ACGUGACAC GUUCGGAGAATT-3' (antisense). Transfection was allowed to proceed for various times and cells were processed for different assays. The siRNA transfection efficiency of 
Lipofectamine RNAiMax in cells was determined by the BLOCK-iT Alexa FluorR Red Fluorescent Oligo protocol (Invitrogen). Significance of differences was assessed using paired one tailed t-test.

Real-time PCR analysis. Total RNA was extracted from glioma specimen or glioma cells using TRIzol reagent (Invitrogen). Real-time quantitative PCR analysis was performed using SYBR Green Master Mix kit on Thermo Fisher connect RealTime PCR platform. In brief, each PCR reaction mixture containing $10 \mu \mathrm{l}$ of $2 \mathrm{X}$ SYBR GreenMaster Mix, $1 \mu \mathrm{l}$ of sense and antisense primers $(5 \mu \mathrm{mol} / \mu \mathrm{l})$ and $1 \mu \mathrm{l}$ of cDNA (10 ng), was run for 40 cycles with denaturation at $95^{\circ} \mathrm{C}$ for $15 \mathrm{sec}$, annealing at $60^{\circ} \mathrm{C}$ for $30 \mathrm{sec}$, and extension at $72^{\circ} \mathrm{C}$ for $30 \mathrm{sec}$, in a total volume of $20 \mu \mathrm{l}$. For relative quantification, $2^{-\Delta \Delta C T}$ was calculated and used as an indication of the relative expression levels, which was calculated by subtracting CT values of the control gene from the CT values of MEG3 and DNMT1. Real-time PCR was carried out under a standard protocol using the following primers: DNMT1 (forward, 5'-CGG CTTCAGCACCTCATTTG-3'; reverse, 5'-AGGTCGAGTCG GAATTGCTC-3'), MEG3 (forward, 5'-ATCATCCGT CCACC TCCTTGTCTTC-3'; reverse, 5'-GTATGAGCATAGCAAAG GTCAGGGC-3'). GAPDH was applied as an internal control. The primer sequences of GAPDH were 5'-AGCAAGAGCACA AGAGGAAG-3' and 5'-GGTTGAGCACAGGGTACTTT-3'.

MTT assay. U251 and U87 glioma cells were trypsinized, resuspended, seeded into a 96-well plate with a concentration of 2,000 cells per well, and incubated at $37^{\circ} \mathrm{C} 3$ days postsiRNA transfection. The number of viable cells was measured at daily intervals (at 12, 24, 48 and $72 \mathrm{~h}$ ). At each time-point, $10 \mu \mathrm{l}$ of $5 \mathrm{mg} / \mathrm{ml}$ MTT (Dingguo Biotechnology) was added, and incubation was continued for $4 \mathrm{~h}$. Then the medium was removed carefully and $150 \mu \mathrm{l}$ of DMSO was added at the end of incubation. The absorbance was measured at $592 \mathrm{~nm}$ on the spectrophotometer.

Colony formation assay. A total of $200 \mathrm{U} 251$ and U87 glioma cells were seeded in 6-well plates after 3 days of siRNA transfection. The medium was changed at regular time intervals. After 11 days of culture at $37^{\circ} \mathrm{C}$, the natural colonies were washed with PBS and fixed with $4 \%$ paraformaldehyde for $30 \mathrm{~min}$ at room temperature. The colonies were then stained with Giemsa for $10 \mathrm{~min}$, washed with water and air-dried. The total number of colonies with $>50$ cells was counted under fluorescence microscopy.

Cell apoptosis assay. For cell apoptosis assay, cells were plated into 6 -well plates at $1 \times 10^{5}$ cells per well. Several days after siRNA transfection or 5-AzadC treatment, the cells were harvested by trypsinization and washed with PBS. Annexin V-fluorescein isothiocyanate and PI double-staining (BD Biosciences) was used to detect and quantify cellular apoptosis by FCM. All the tests were performed in triplicate.

Western blotting. U251 and U87 glioma cells were lysed with RIPA lysis buffer (Beyotime, China). Whole extracts were prepared, and protein concentrations were determined using the BCA protein assay kit (Boster). Whole-cell extracts (20 or
$40 \mu \mathrm{g}$ ) were then fractionated by electrophoresis through an 8 or $12 \%$ sodium dodecyl sulfate-polyacrylamide gel electrophoresis (SDS-PAGE). Gels were run at a $120 \mathrm{~V}$ for $2 \mathrm{~h}$ before transfer onto a PVDF membrane (Millipore Corp., Billerica, MA, USA). After blocking against non-specific protein binding, nitrocellulose blots were incubated for $1 \mathrm{~h}$ with primary antibodies diluted in TBS/Tween-20 $(0.075 \%$ Tween-20) containing 3\% Marvel. Anti-DNMT1, anti-MDM2 and anti-p53 were diluted 1:400. Following incubation with the primary antibody, blots were washed three times in TBS/ Tween-20 before incubation for $1 \mathrm{~h}$ with goat anti-mouse or mouse anti-rabbit horseradish peroxidase conjugated antibody at a 1:10,000 dilution in TBS/Tween-20 containing 5\% milk. After extensive washing in TBS/Tween-20, the blots were rinsed with distilled water and proteins were detected using the enhanced chemiluminescence system. Proteins were visualized with ECL-chemiluminescent kit (ECL-plus, Thermo Scientific).

Statistical analysis. The data are expressed as mean \pm SD of three independent experiments. Statistical analysis was performed using the Student's t-test and the one-way analysis of variance (ANOVA). Pearson's test was performed to calculate the association between DNMT1 and MEG3 expression. Significance was defined as $\mathrm{P}<0.05$.

\section{Results}

MEG3 methylation status and loss of MEG3 expression in glioma. To determine the expression levels of MEG3 in glioma samples and cell lines, total RNAs were extracted from glioma tissues at low-grade (grade I and II), high-grade (grade III and IV), and normal brain tissue samples, and the expression level of MEG3 was analyzed using qRT-PCR. As shown in Fig. 1A, the levels of MEG3 expression were significantly decreased in glioma tissues compared with normal brain tissues. To explore whether there is any association between the downregulation of MEG3 and the pathogenesis of glioma, we detected the expression of MEG3 in glioma samples with different grades and found marked downregulation in high-grade glioma samples and to a lesser degree, decreased in low-grade glioma samples, as compared to normal brain tissues, which gave clues that MEG3 may be involved in the disease pathogenesis in glioma (Fig. 1B). Of note, 3 glioma cell lines displayed significantly downregulated expression of MEG3 relative to that in normal brain tissues (Fig. 1C).

To investigate the mechanism underlying the decreased MEG3 expression, we analyzed whether MEG3 promoter region hypermethylation is responsible for the downregulation of MEG3 expression. Methylation-specific PCR analysis indicated that the promoter region of MEG3 from glioma tissues in which MEG3 expression was downregulated was strongly methylated, whereas normal brain tissues and low-grade glioma tissues in which MEG3 expression was present had unmethylated MEG3 promoter region (Fig. 1D). These results suggest that methylation of MEG3 promoter likely contributes to the loss of MEG3 expression in high-grade glioma.

MEG3 downregulation due to the hypermethylation of its promoter region is restored after treatment of glioma cells 

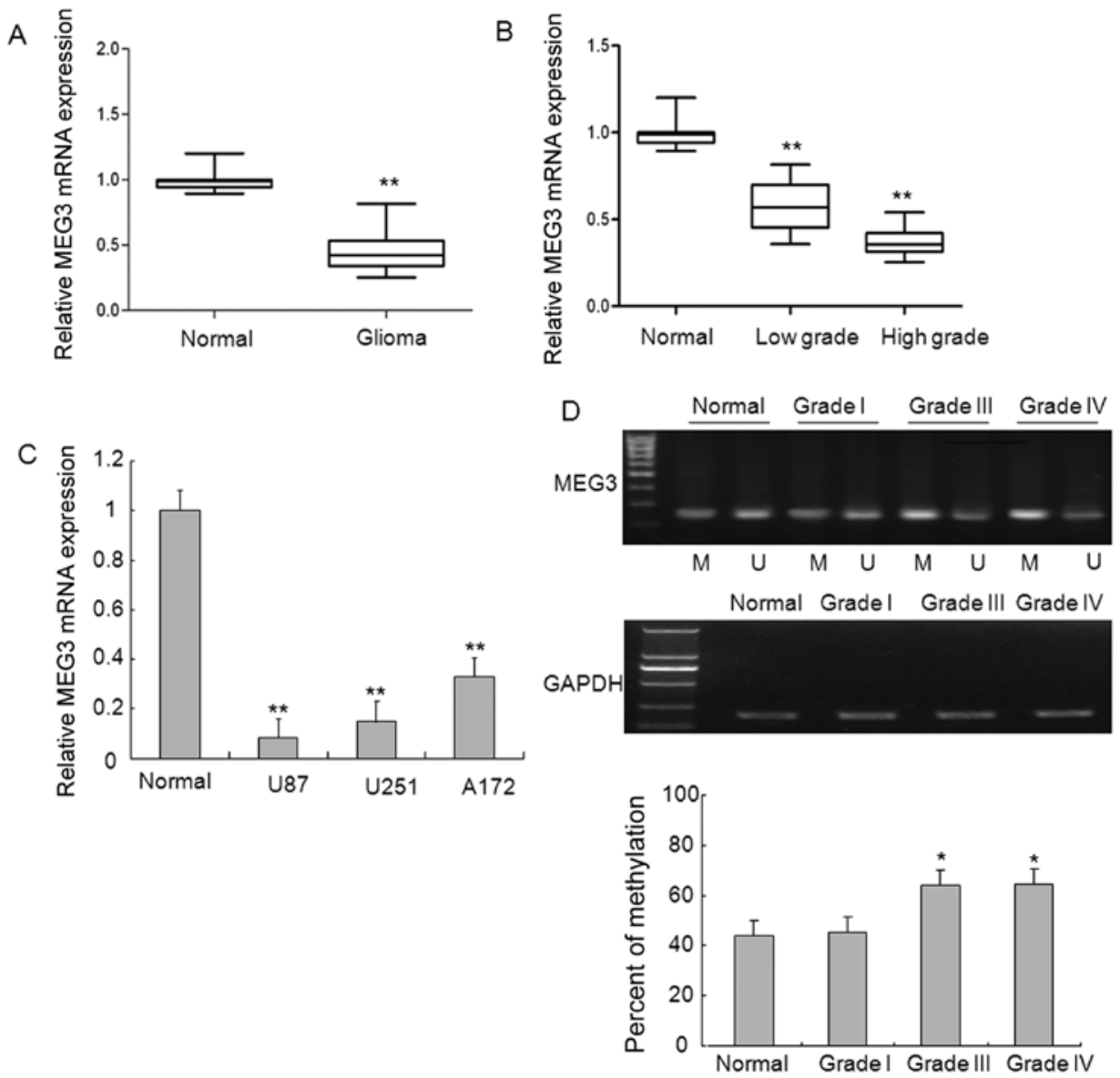

Figure 1. Methylation of MEG3 promoter and downregulation of MEG3 in human glioma samples and cells lines. (A) MEG3 mRNA expression was decreased in glioma samples. (B) Increased malignancy of glioma samples was correlated with decreased MEG3 mRNA expression. (C) The mRNA level of MEG3 in different glioma cells and normal brain tissues. (D) MEG3 hypermethylation was observed in human glioma samples with different malignant grades. Each bar represents the mean $\pm \mathrm{SD}$ of three independent experiments performed in duplicate. ${ }^{* *} \mathrm{P}<0.01$ vs. normal control.
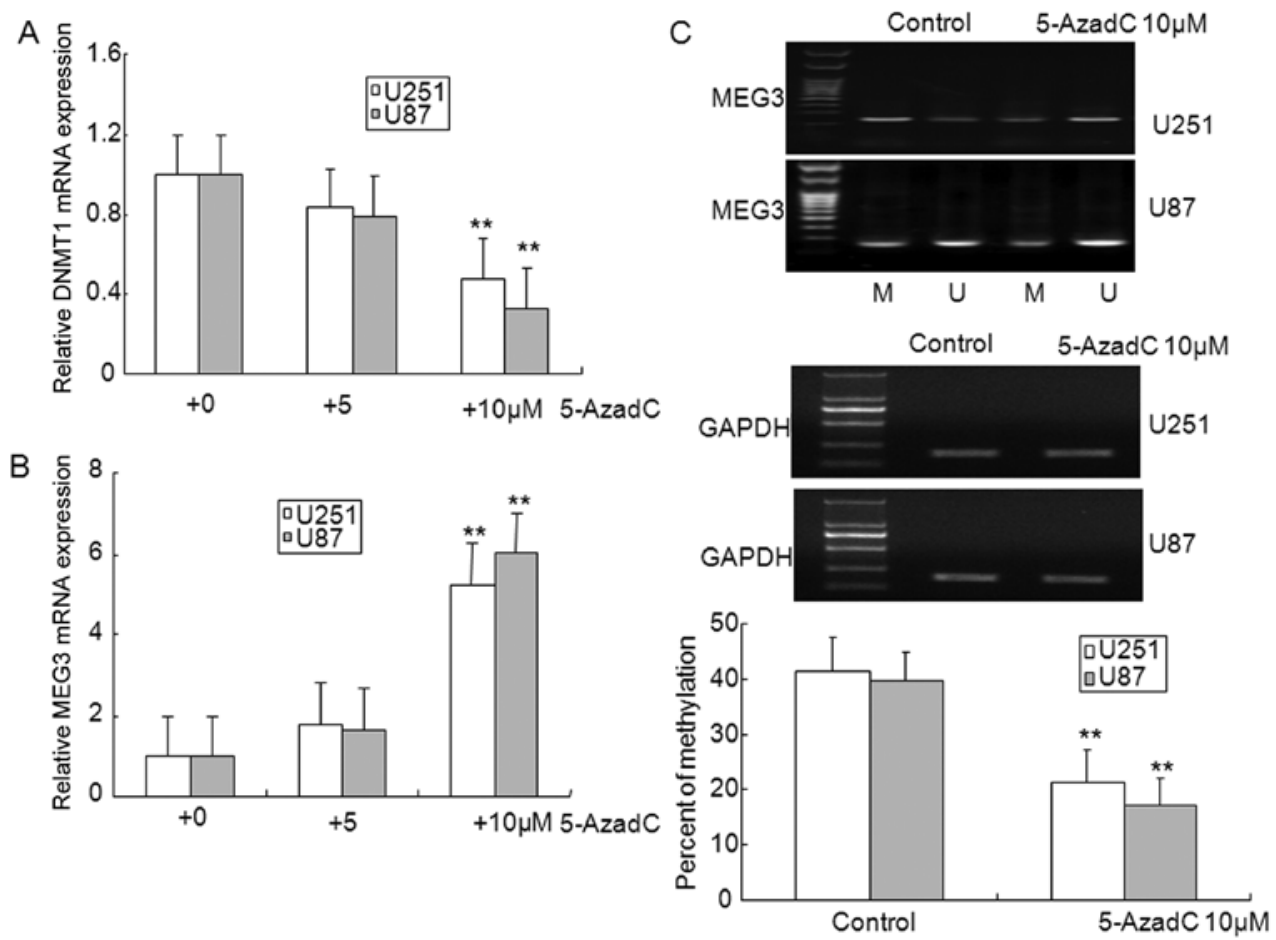

Figure 2. 5-AzadC treatment induced MEG3 promoter demethylation and MEG3 expression in U251 and U87 glioma cells. (A and B) 5-AzadC treatment inhibited the expression of DNMT1 and upregulated the MEG3 expression. (C) MEG3 promoter demethylation was induced in glioma cells challenged with 5 -AzadC. Each bar represents the mean \pm SD of three independent experiments performed in duplicate. ${ }^{* *} \mathrm{p}<0.01 \mathrm{vs}$. control. 


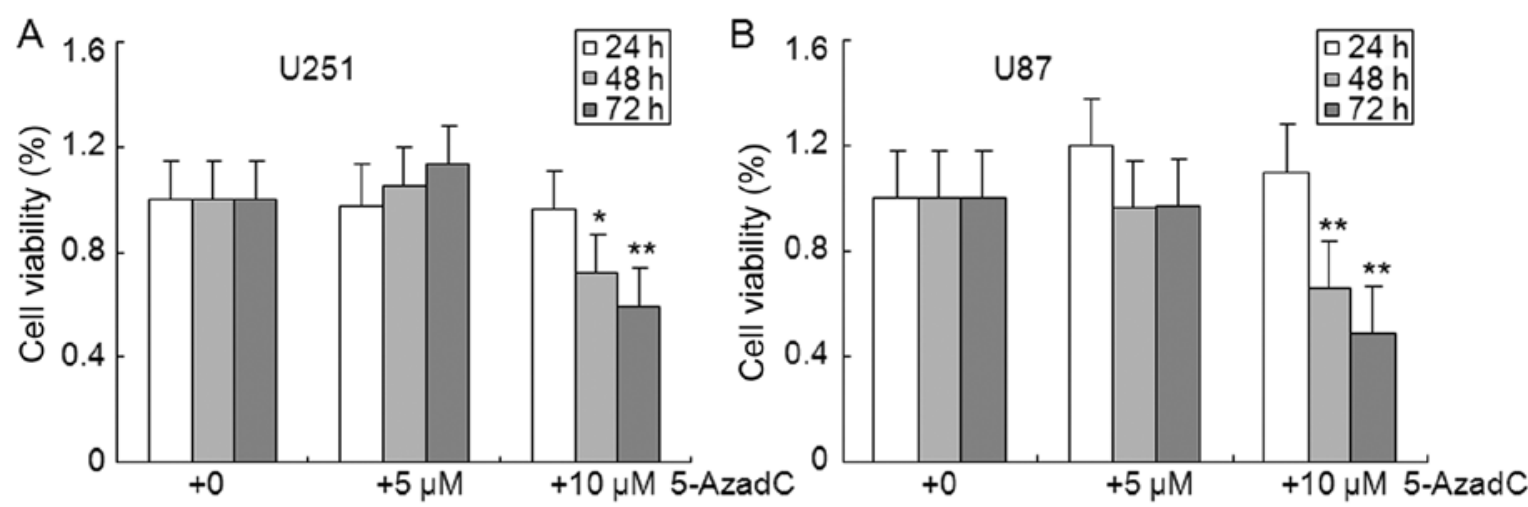

C

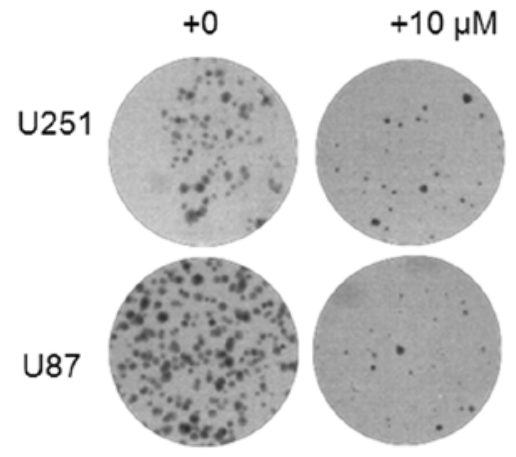

5-AzadC
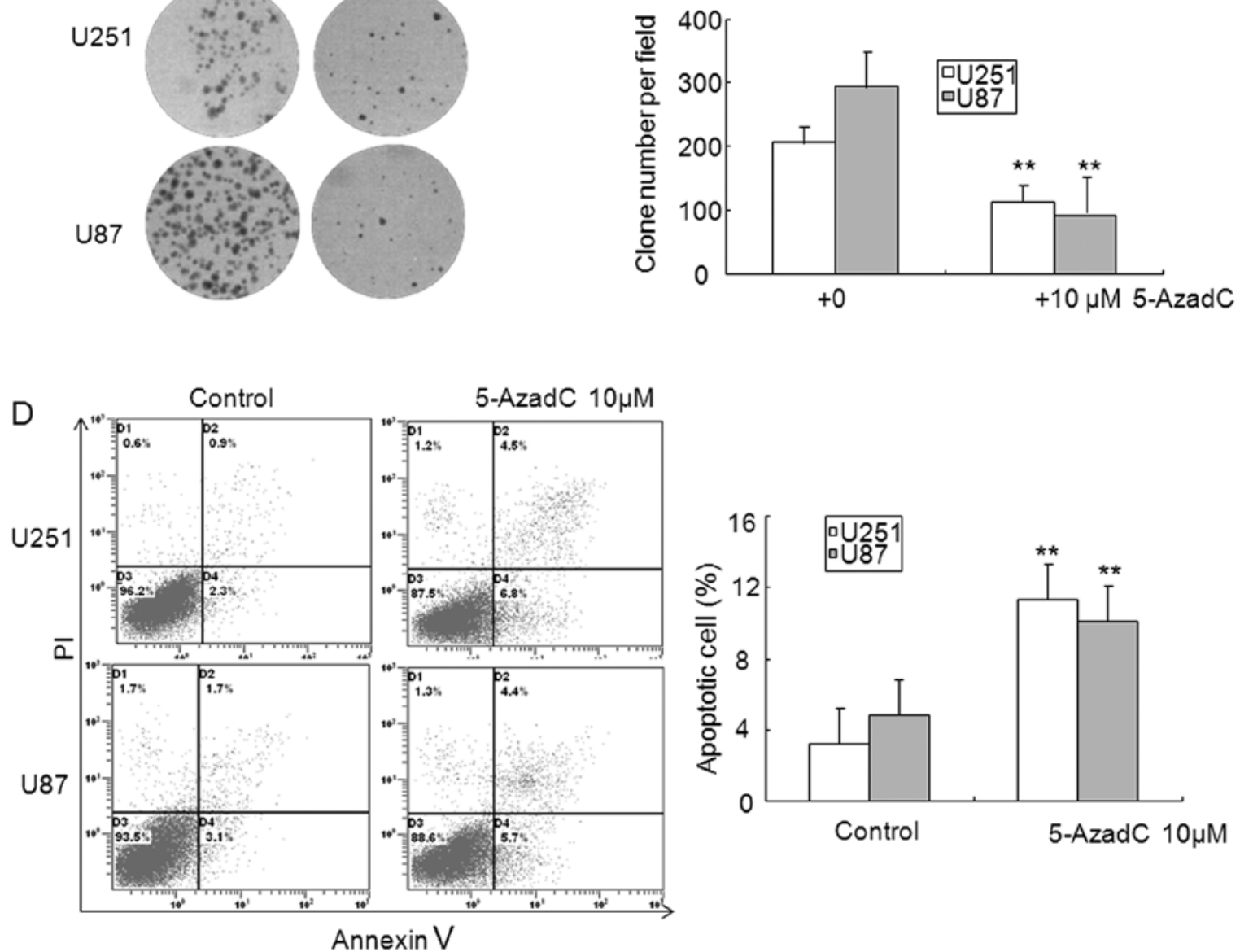

Figure 3. 5-AzadC treatment inhibits cell proliferation and induces cells apoptosis in U251 and U87 glioma cells. (A) 5-AzadC treatment inhibited cell viability in glioma cells. (B) 5-AzadC treatment reduced clone formation in glioma cells. (C) The apoptotic glioma cells (both the Annexin V-positive/PI-negative cells and the Annexin V-positive/PI-positive population) were increased upon 5-AzadC treatment. The data represent the mean \pm SD of three different experiments. ${ }^{*} \mathrm{p}<0.05$ vs. control, ${ }^{* *} \mathrm{p}<0.01$ vs. control.

with the DNMT inhibitor 5-AzadC. To further confirm that the loss of MEG3 expression was caused by promoter methylation in U87 and U251 glioma cells in vitro, the DNMT inhibitor 5-AzadC was used to inhibit methylation. As illustrated in Fig. 2A and B, treatment with $10 \mu \mathrm{M}$ 5-AzadC markedly inhibited the expression of DNMT1, and upregulated the expression of MEG3, however, expression of DNMT1 and MEG3 did not show significant changes in $5 \mu \mathrm{M}$ 5-AzadC treated glioma cells. In addition, aberrant hypermethylation of MEG3 was diminished in 5-AzadC treated glioma cells compared with control cells (Fig. 2C). The MTT assay showed that exposure with 5-AzadC for 48 and $72 \mathrm{~h}$ significantly inhibited the cell viability of U251 and U87 glioma cells (Fig. 3A and B). The clone formation assay was performed to further confirm the effect of 5-AzadC on glioma cell proliferation, and data indicated that 5-AzadC treatment significantly inhibited the number of clones in U251 and U87 glioma cells (Fig. 3C). Reduction 
A

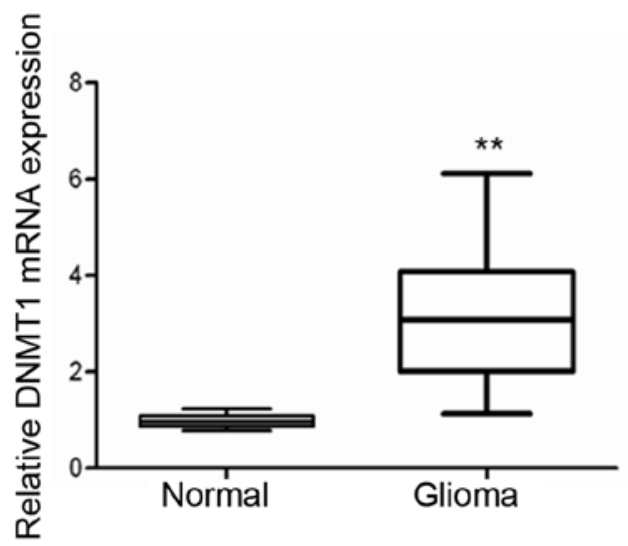

B

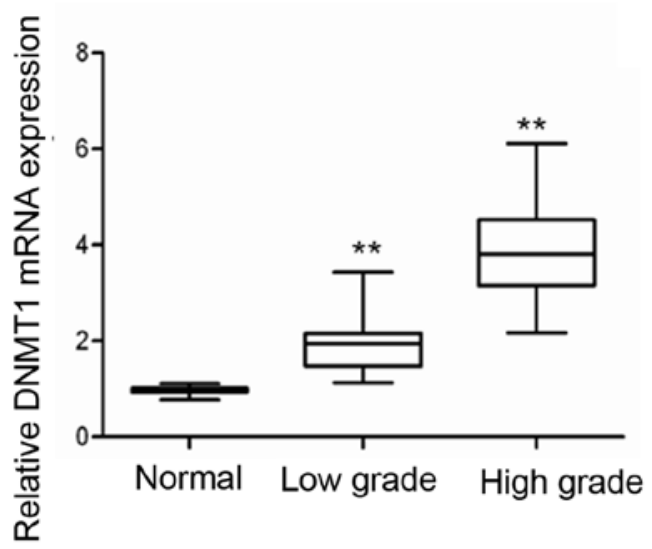

C

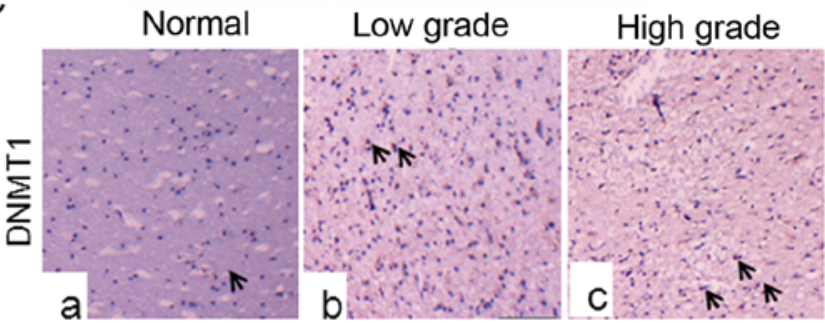

$\mathrm{D}$

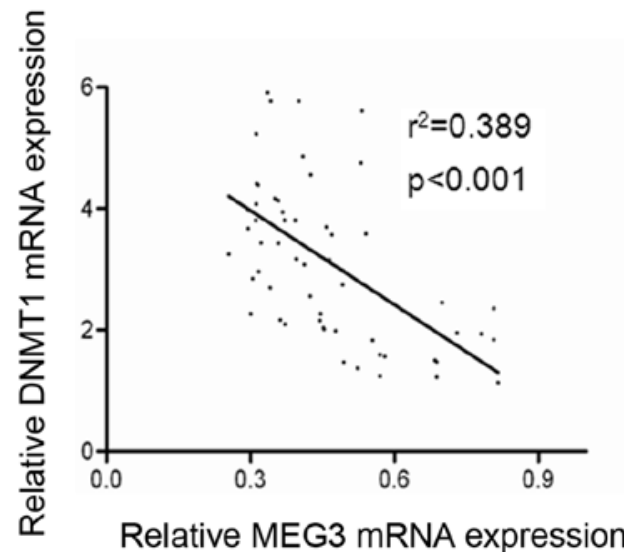

Figure 4. DNMT1 is overexpressed and negatively correlated with MEG3 expression in human glioma samples. (A) DNMT1 mRNA expression was upregulated in glioma samples. (B) Increased mRNA expression of DNMT1 was associated with malignant grades in human glioma samples. (C) The positive cells of DNMT1 were increased in malignant glioma specimens. Glioma specimens showed nucleus staining $(\mathrm{b}$ and $\mathrm{c}$ ) and normal brain tissues showed negative expression (a). Magnification, $50 \mu \mathrm{m}$. (D) DNMT1 mRNA expression was negatively correlated with MEG3 mRNA expression in 55 glioma samples. ${ }^{* *} \mathrm{P}<0.01$ vs. normal control.

of glioma cell growth by 5 -AzadC treatment suggests that these cells may undergo programmed cell death. To test this hypothesis, we analyzed cell apoptosis induced by 5-AzadC using Annexin V and PI double staining. As shown in Fig. 3D, 5-AzadC treatment significantly induced apoptosis of U251 and U87 glioma cells compare to control cells. These results suggest that the decreased expression of MEG3 is related to reversible epigenetic mechanisms such as DNA promoter region methylation in glioma.

DNMT1 is involved in hypermethylation of MEG3 in glioma cells. To gain insights into the possible involvement of DNMT1 in glioma, we examined the expression of DNMT1 in glioma tissues. As shown in Fig. 4A, the levels of DNMT1 mRNA expression were significantly upregulated in gliomas tissues compared with normal brain tissues. The levels of DNMT1 mRNA expression were positively correlated to glioma grades, with lower grades exhibiting upregulation $>3$-fold and higher grades demonstrating a $>5$-fold increase in DNMT1 expression (Fig. 4B). These data provided us with experimental evidence that DNMT1 expression was upregulated in a graded manner in gliomas, with higher grades showing highest expression. In addition, immunohistochemical analyses showed that DNMT1 expression was significantly increased in high-grade glioma, and low-grade glioma compared with normal brain tissues, suggesting this gene is associated with glioma malignancy (Fig. 4C). Next, we investigated whether levels of DNMT1 mRNA expression was inversely correlated with MEG3 expression in glioma tissues. A statistically significant inverse correlation was observed between DNMT1 mRNA and MEG3 (Fig. 4D), supporting the role of DNMT1 in expression of MEG3.

To determine the role of DNMT1 in glioma, the expression of DNMT1 was inhibited by DNMT1 RNAi. Data from the MTT assay showed that transfection with DNMT1 RNAi, but not scrambled RNAi for 48 and $72 \mathrm{~h}$ reduced the cell viability of U251 and U87 glioma cells (Fig. 5A and B). The results from the clone formation assay verified the MTT results, and the finding that DNMT1 RNAi inhibited clone formation in U251 and U87 glioma cells (Fig. 5C). The apoptosis of glioma cells was also observed in DNMT1 knockdown U251 and U87 glioma cells (Fig. 5D). In addition, DNMT1 knockdown in U251 and U87 glioma cells ameliorated MEG3 methylation, and restored MEG3 mRNA expression (Fig. 6A-C). These results suggest that MEG3 hypermethylation was dependent on DNMT1 in glioma. 

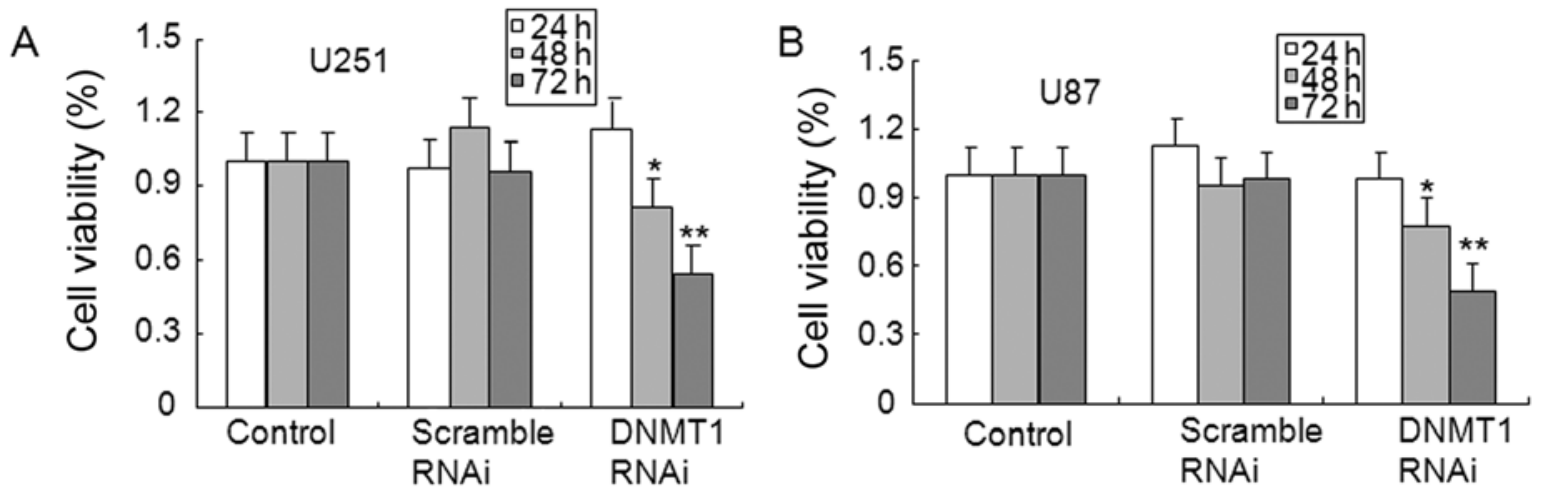

C
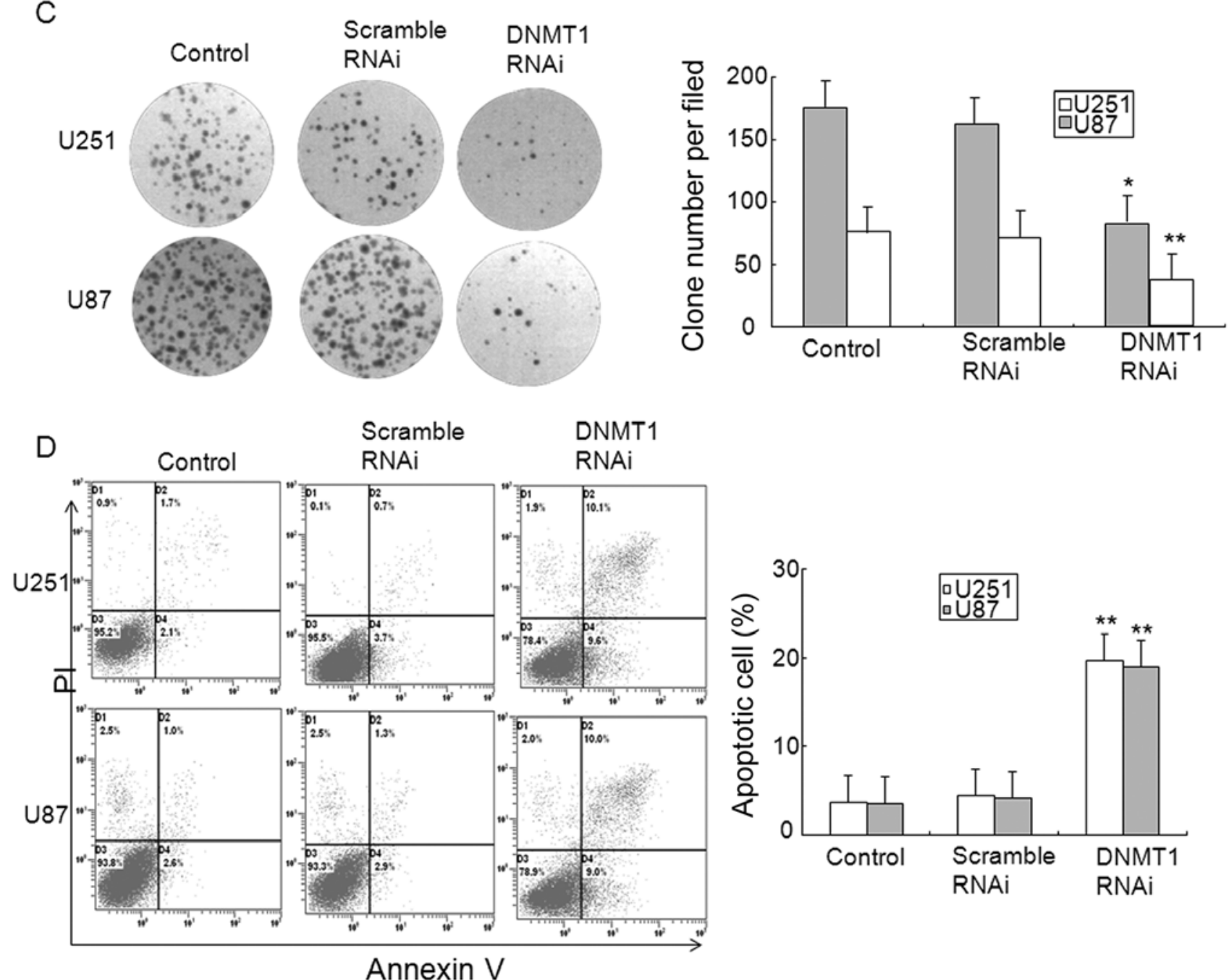

Figure 5. DNMT1 knockdown inhibits cell proliferation and induced cells apoptosis in U251 and U87 glioma cells. (A and B) Glioma cell viability was inhibited after transfection with DNMT1 siRNA. (C) Knockdown of DNMT1 inhibited clone formation in glioma cells. (D) The apoptotic cells (both the Annexin V-positive/PI-negative cells and the Annexin V-positive/PI-positive population) were increased in glioma cells upon inhibition of DNMT1. The data represent the mean $\pm \mathrm{SD}$ of three different experiments. ${ }^{*} \mathrm{p}<0.05,{ }^{* *} \mathrm{p}<0.01$ vs. scramble RNAi.

The downregulation of MEG3 due to hypermethylation of MEG3 contributes to the inhibition of p53 pathway in glioma cells. To study the potential signaling pathways affected by MEG3 methylation, the protein levels of both MDM2 and p53 were examined in glioma cells. As illustrated in Fig. 7A, treatment with 5-AzadC reduced the expression levels of DNMT1 and MDM2 proteins in U251 and U87 glioma cells, whereas upregulated the levels of p53 protein expression. Of note, the repression of MDM2 expression mediated by MEG3 may be correlated with enhanced expression of p53 protein.

Finally, the effect of DNMT1 silencing on the activation of p53 pathway was examined in glioma cells. As illustrated in Fig. 7B, knockdown of DNMT1 significantly repressed the expression of DNMT1 and MDM2 protein compared to 
A

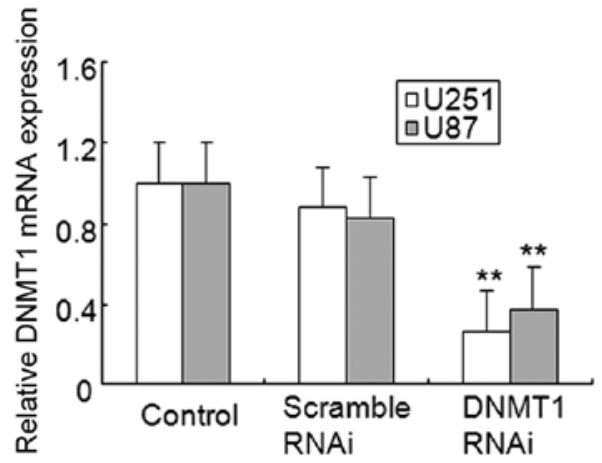

C Control Scramble DNMT1
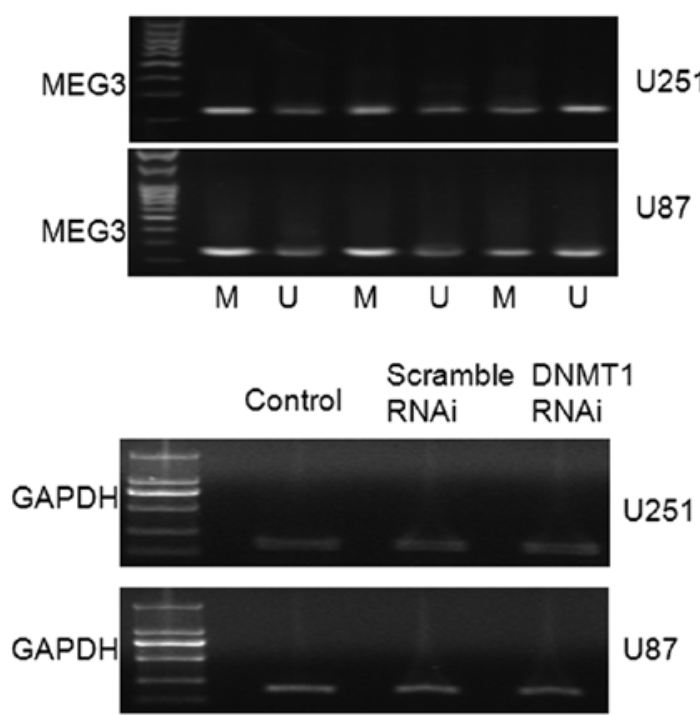

$\mathrm{B}$

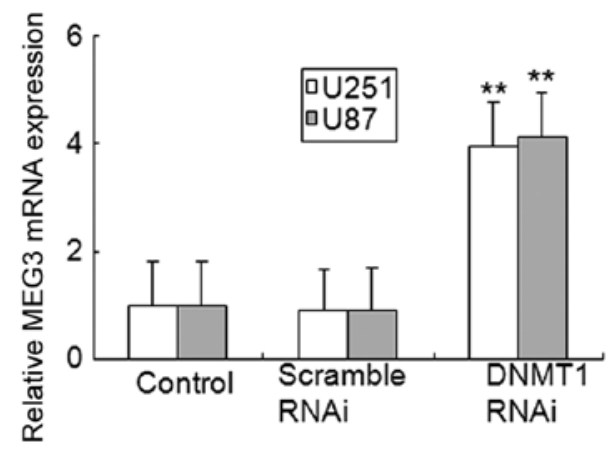

Figure 6. DNMT1 knockdown induces MEG3 promoter demethylation and MEG3 expression in U251 and U87 glioma cells. (A and B) Knockdown of DNMT1 inhibited the mRNA level of DNMT1 and upregulated MEG3 mRNA expression in glioma cells. (C) MEG3 promoter demethylation was observed in glioma cells with DNMT1 siRNA transfection. Each bar represents the mean \pm SD of three independent experiments performed in duplicate. ${ }^{* *}$ p $<0.01$ vs. scramble RNAi.

control or scrambled U251 and U87 glioma cells, however, the expression levels of p53 protein were induced. These results suggest that an aberrant methylation occurred in MEG3 promoter contributes to the decrease in p53 expression by the activation of MDM2 in gliomas.

\section{Discussion}

Malignant glioma is the most common and fatal brain tumor world-wide (18). In addition to conventional therapeutic strategies, targeted therapies are currently identified to interfere with the transduction of pivotal signaling pathways or to suppress the function of tumor specific molecules in malignant glioma. It is widely accepted that the future treatment options for glioma will greatly benefit from our improved understanding of the complex molecular mechanism in gliomas.

Maternally expressed gene 3 (MEG3), an imprinted gene, is a tumor suppressor gene located in chromosome $14 \mathrm{q} 32$ (19). MEG3 is downregulated in a number of cancer types, suggesting that MEG3 is a potential tumor suppressor gene. In the present study, our findings revealed that MEG3 expression was markedly decreased in glioma tissues, and several glioma cell types compared with normal brain tissues. Loss of MEG3 expression can result from epigenetic modification, such as microRNA or promoter hypermethylation $(20,21)$. Hypermethylation mostly occurs at the promoter of genes that are involved in processes resulting in tumor formation and progression and has been shown for diverse types of genes related to tumor suppression, DNA repair, cell cycle regulation, apoptosis, invasion, and migration. Recently, several reports have shown that loss of MEG3 expression and promoter hypermethylation of MEG3 were observed in several types of human tumors, including pituitary adenomas, neuroblastomas, pheochromocytomas, Wilms tumors, and other carcinomas $(12,22)$. In the present study, marked hypermethylation of MEG3 and the loss of MEG3 expression was found in high grade gliomas. These results suggest that the downregulation of MEG3 expression probably attributed, at least in part, to epigenetic modification by the hypermethylation of MEG3 promoter in gliomas in vivo.

5 -AzadC is a nucleoside analog of cytidine in which the 5 -carbon of the pyrimidine ring is replaced by a nitrogen. Following incorporation into DNA, 5-AzadC covalently binds to DNMTs through the nitrogen in the 5-position of the 
A
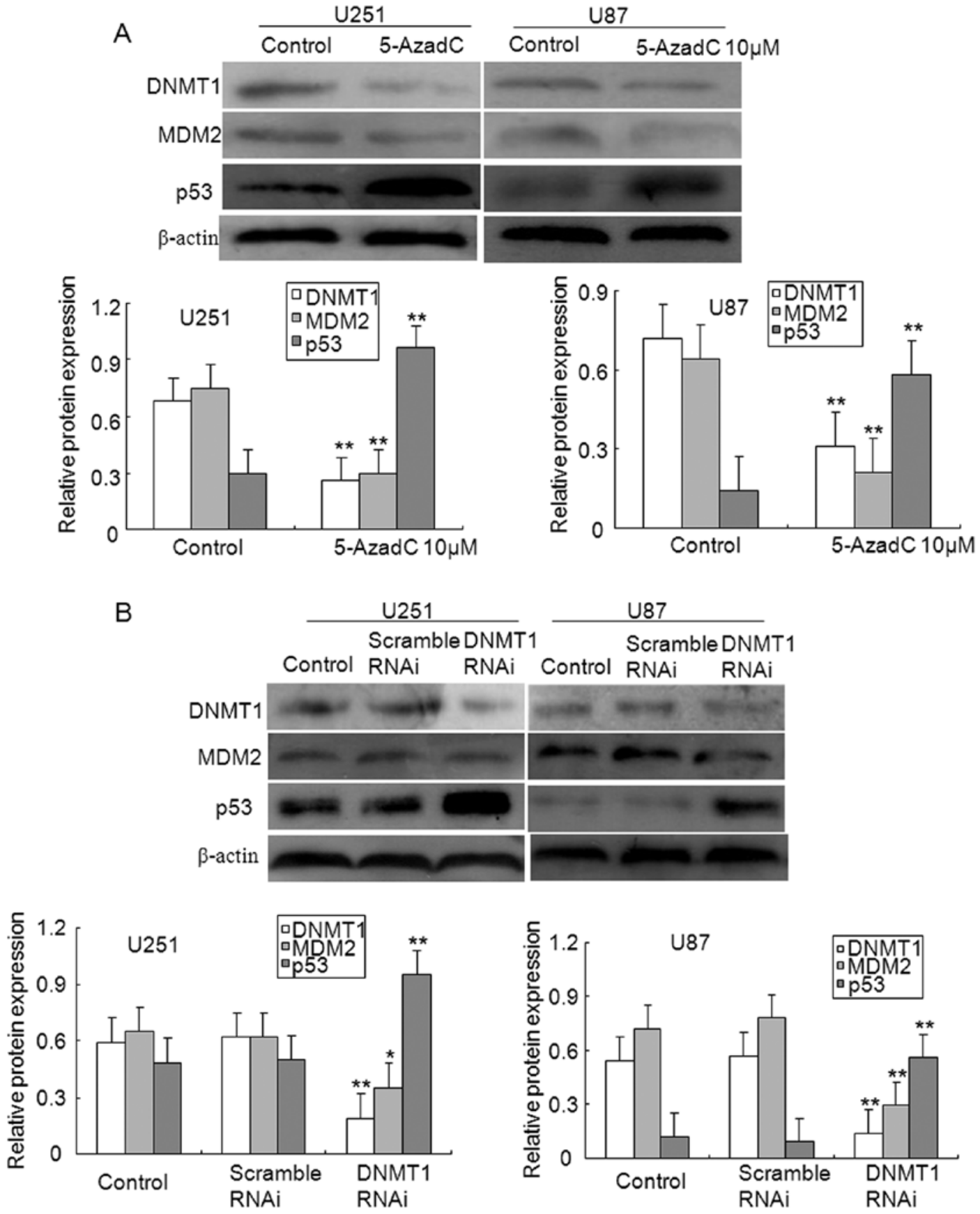

Figure 7. Epigenetic silencing of MEG3 represses the p53 pathway in U251 and U87 glioma cells. (A) 5-AzadC treatment inhibited the levels of DNMT1 and MDM2 protein expression and upregulated p53 protein expression in glioma cells. (B) DNMT1 knockdown inhibited the levels of DNMT1 and MDM2, and upregulated p53 protein expression in glioma cells. Each sample was analyzed in triplicate and $\beta$-actin was used as an internal control. ${ }^{* *}$ p $<0.01$ vs. control or scramble RNAi.

modified pyrimidine and traps it for proteosomal degradation, leading to inhibition of the activity of the maintenance DNA methyltransferase DNMT1 $(23,24)$. A recent study by Tsai et al showed that treatment of cancer cells with DNMT inhibitors resulted in sustained changes in gene expression and critical signaling pathways involved in tumorigenesis (25). Our present results showed that treatment of glioma cells with 5-AzadC reversed the methylation of the MEG3, and restored MEG3 expression. Zhang et al reported that overexpression of MEG3 suppresses DNA synthesis, colony formation in meningioma cells (21). In addition, forced expression of MEG3 inhibited the proliferation and induced apoptosis in glioma cells (26). In the present study, we demonstrated that 5-AzadC treatment inhibited the growth of glioma cells, and resulted in apoptosis of glioma cells. These findings suggest that 5-AzadC inhibits hypermethylation of MEG3 and subsequently induces MEG3 expression, followed by blocking the growth and inducing the apoptosis of glioma cells.

DNA methylation involves transfer of a methyl group to the 5-carbon in cytosine of a $\mathrm{CpG}$ dinucleotide by DNA methyltransferases (DNMTs) - DNMT1, DNMT3A, and DNMT3B. DNMT1 is the main enzyme responsible for maintaining the 
methylation pattern onto daughter strands after DNA replication (27). DNMT1 is frequently upregulated in various human cancers such as hepatic, prostate, and gliomas (28-30). In this study, we found that the upregulation of DNMT1 expression associated with gliomas malignancy is observed in gliomas specimens. Selective depletion of DNMT1 has been shown to induce demethylation of promoters and re-expression of the silenced genes, including tumor suppressor genes or tumor suppressive miRNAs, due to hypermethylation $(31,32)$. Here, we demonstrated that knockdown of DNMT1 inhibited the growth of glioma cells and induced apoptosis of glioma cells, and reversed the methylation of MEG promoter and subsequently restored MEG3 expression. These findings suggest that the upregulation of DNMT1 promotes MEG3 hypermethylation with the downregulation of MEG3 expression, followed by promotion of the growth of glioma cells.

Tumor suppression is a cellular defense mechanism preventing the neoplastic transformation of normal cells, and the tumor suppressor p53 has a central role in the initiation and progression of tumors (33). P53 levels are drastically lowered due to rapid degradation via the ubiquitin-proteasome pathway. The ubiquitination of p53 is mainly regulated by MDM2, referred to as an E3 ubiquitin ligase. A decrease in MDM2 protein level was observed in non-small cell lung cancer cells transfected with MEG3 (34). Overexpression of MEG3 resulted in a significant increase in p53 protein levels and dstrongly stimulated p53-dependent transcription. Moreover, MDM2 levels were downregulated in cells transfected with MEG3, suggesting that MDM2 suppression contributes, at least in part, to p53 accumulation induced by MEG3 (14). In the present report, we provide two lines of evidence suggesting that epigenetic silencing of MEG3 results in the repression of p53 signal pathways and subsequently regulates the growth of glioma cells. First, the inhibition of DNMT1 by 5-AzadC, or its specific downregulation by siRNA reversed the MEG3 promoter methylation and restored MEG3 expression. Second, treatment with 5-AzadC or knockdown of DNMT1 repressed the expression of MDM2 and induced the activation of $\mathrm{p} 53$ pathways. MEG3 is known to suppress the activation of MDM2 pathways, and following the activation of p53 pathway. Therefore, the downregulation of MEG3 expression in glioma cells results from the occurrence of aberrant hypermethylation in the MEG3 promoter. Such downregulation of MEG3 contributes to MDM2 pathways, and subsequently represses the p53 pathway.

In conclusion, our findings from the present study strongly suggest MEG3 as a candidate tumor suppressor gene associated with the pathogenesis and progression of human gliomas. The expression of MEG3 could be restored via epigenetic modification, which may suggest therapeutic potential for the treatment of gliomas. To our knowledge, this is the first report on hypermethylation of MEG3 as a mechanism of the development of gliomas. MEG3/Gt12 is a single-copy gene, these isoform expression patterns are tissue and cell type specific. Twelve different MEG3 gene transcripts were generated by alternative splicing, using different exons in the middle of the RNA (35). Further investigation of the structure-function relationship of MEG3 is necessary to provide more information associated with the pathogenesis of human gliomas to reveal novel mechanisms to broaden our knowledge of the involvement of non-coding RNAs in human gliomas biology, and eventually to develop new therapeutic strategies for gliomas.

\section{Acknowledgements}

This study was supported by the National Natural Science Foundation of China (no. 81402078), Natural Science Foundation of Anhui Province (no. 1508085MH194).

\section{References}

1. Wang $\mathrm{Y}$ and Jiang $\mathrm{T}$ : Understanding high grade glioma: Molecular mechanism, therapy and comprehensive management. Cancer Lett 331: 139-146, 2013.

2. Stupp R, Hegi ME, Mason WP, van den Bent MJ, Taphoorn MJ, Janzer RC, Ludwin SK, Allgeier A, Fisher B, Belanger K, et al; European Organisation for Research and Treatment of Cancer Brain Tumour and Radiation Oncology Groups; National Cancer Institute of Canada Clinical Trials Group: Effects of radiotherapy with concomitant and adjuvant temozolomide versus radiotherapy alone on survival in glioblastoma in a randomised phase III study: 5-year analysis of the EORTC-NCIC trial. Lancet Oncol 10: 459-466, 2009.

3. Wen PY and Kesari S: Malignant gliomas in adults. N Engl J Med 359: 492-507, 2008.

4. Wilusz JE, Sunwoo H and Spector DL: Long noncoding RNAs: Functional surprises from the RNA world. Genes Dev 23: 1494-1504, 2009.

5. Nagano $T$ and Fraser P: No-nonsense functions for long noncoding RNAs. Cell 145: 178-181, 2011.

6. Wu Y, Zhang L, Zhang L, Wang Y, Li H, Ren X, Wei F, Yu W, Liu T, Wang X, et al: Long non-coding RNA HOTAIR promotes tumor cell invasion and metastasis by recruiting EZH2 and repressing E-cadherin in oral squamous cell carcinoma. Int $\mathbf{J}$ Oncol 46: 2586-2594, 2015.

7. Wang Y, Chen W, Yang C, Wu W, Wu S, Qin X and Li X: Long non-coding RNA UCA1a (CUDR) promotes proliferation and tumorigenesis of bladder cancer. Int J Oncol 41: 276-284, 2012.

8. Ørom UA, Derrien T, Beringer M, Gumireddy K, Gardini A, Bussotti G, Lai F, Zytnicki M, Notredame C, Huang Q, et al: Long noncoding RNAs with enhancer-like function in human cells. Cell 143: 46-58, 2010.

9. Tian D, Sun S and Lee JT: The long noncoding RNA, Jpx, is a molecular switch for X chromosome inactivation. Cell 143: 390-403, 2010.

10. Kobayashi S, Wagatsuma H, Ono R, Ichikawa H, Yamazaki M, Tashiro H, Aisaka K, Miyoshi N, Kohda T, Ogura A, et al: Mouse Peg9/Dlk1 and human PEG9/DLK1 are paternally expressed imprinted genes closely located to the maternally expressed imprinted genes: Mouse Meg3/Gt12 and human MEG3. Genes Cells 5: 1029-1037, 2000.

11. Anwar SL, Krech T, Hasemeier B, Schipper E, Schweitzer N, Vogel A, Kreipe H and Lehmann U: Loss of imprinting and allelic switching at the DLK1-MEG3 locus in human hepatocellular carcinoma. PLoS One 7: e49462, 2012.

12. Astuti D, Latif F, Wagner K, Gentle D, Cooper WN, Catchpoole D, Grundy R, Ferguson-Smith AC and Maher ER: Epigenetic alteration at the DLK1-GTL2 imprinted domain in human neoplasia: Analysis of neuroblastoma, phaeochromocytoma and Wilms' tumour. Br J Cancer 92: 1574-1580, 2005.

13. Gejman R, Batista DL, Zhong Y, Zhou Y,Zhang X, Swearingen B, Stratakis CA, Hedley-Whyte ET and Klibanski A: Selective loss of MEG3 expression and intergenic differentially methylated region hypermethylation in the MEG3/DLK1 locus in human clinically nonfunctioning pituitary adenomas. J Clin Endocrinol Metab 93: 4119-4125, 2008.

14. Zhou Y, Zhong Y, Wang Y, Zhang X, Batista DL, Gejman R, Ansell PJ, Zhao J, Weng C and Klibanski A: Activation of p53 by MEG3 non-coding RNA. J Biol Chem 282: 24731-24742, 2007.

15. Sheng X, Li J, Yang L, Chen Z, Zhao Q, Tan L, Zhou Y and Li J: Promoter hypermethylation influences the suppressive role of maternally expressed 3, a long non-coding RNA, in the development of epithelial ovarian cancer. Oncol Rep 32: 277-285, 2014. 
16. Hervouet E, Vallette FM and Cartron PF: Impact of the DNA methyltransferases expression on the methylation status of apoptosis-associated genes in glioblastoma multiforme. Cell Death Dis 1: e8, 2010

17. Braconi C, Kogure T, Valeri N, Huang N, Nuovo G, Costinean S, Negrini M, Miotto E, Croce CM and Patel T: microRNA-29 can regulate expression of the long non-coding RNA gene MEG3 in hepatocellular cancer. Oncogene 30: 4750-4756, 2011.

18. Furnari FB, Fenton T, Bachoo RM, Mukasa A, Stommel JM, Stegh A, Hahn WC, Ligon KL, Louis DN, Brennan C, et al: Malignant astrocytic glioma: Genetics, biology, and paths to treatment. Genes Dev 21: 2683-2710, 2007.

19. Zhou Y, Zhang X and Klibanski A: MEG3 noncoding RNA: A tumor suppressor. J Mol Endocrinol 48: R45-R53, 2012.

20. Yan J, Guo X, Xia J, Shan T, Gu C, Liang Z, Zhao W and Jin S: MiR-148a regulates MEG3 in gastric cancer by targeting DNA methyltransferase 1. Med Oncol 31: 879, 2014.

21. Zhang X, Gejman R, Mahta A, Zhong Y, Rice KA, Zhou Y, Cheunsuchon P, Louis DN and Klibanski A: Maternally expressed gene 3, an imprinted noncoding RNA gene, is associated with meningioma pathogenesis and progression. Cancer Res 70: 2350-2358, 2010.

22. Zhao J, Dahle D, Zhou Y, Zhang X and Klibanski A: Hypermethylation of the promoter region is associated with the loss of MEG3 gene expression in human pituitary tumors. J Clin Endocrinol Metab 90: 2179-2186, 2005.

23. Kuo HK, Griffith JD and Kreuzer KN: 5-Azacytidine induced methyltransferase-DNA adducts block DNA replication in vivo. Cancer Res 67: 8248-8254, 2007.

24. Lengauer C, Kinzler KW and Vogelstein B: DNA methylation and genetic instability in colorectal cancer cells. Proc Natl Acad Sci USA 94: 2545-2550, 1997.

25. Tsai HC, Li H, Van Neste L, Cai Y, Robert C, Rassool FV, Shin JJ, Harbom KM, Beaty R, Pappou E, et al: Transient low doses of DNA-demethylating agents exert durable antitumor effects on hematological and epithelial tumor cells. Cancer Cell 21: 430-446, 2012.

26. Wang P, Ren $Z$ and Sun P: Overexpression of the long noncoding RNA MEG3 impairs in vitro glioma cell proliferation. J Cell Biochem 113: 1868-1874, 2012.
27. Jurkowska RZ, Jurkowski TP and Jeltsch A: Structure and function of mammalian DNA methyltransferases. ChemBioChem 12: 206-222, 2011

28. Kreth S, Thon N, Eigenbrod S, Lutz J, Ledderose C, Egensperger R, Tonn JC, Kretzschmar HA, Hinske LC and Kreth FW: O-methylguanine-DNA methyltransferase (MGMT) mRNA expression predicts outcome in malignant glioma independent of MGMT promoter methylation. PLoS One 6: e17156, 2011.

29. Nakagawa T, Kanai Y, Ushijima S, Kitamura T, Kakizoe T and Hirohashi S: DNA hypermethylation on multiple $\mathrm{CpG}$ islands associated with increased DNA methyltransferase DNMT1 protein expression during multistage urothelial carcinogenesis. J Urol 173: 1767-1771, 2005.

30. Saito Y, Kanai Y, Nakagawa T, Sakamoto M, Saito H, Ishii H and Hirohashi S: Increased protein expression of DNA methyltransferase (DNMT) 1 is significantly correlated with the malignant potential and poor prognosis of human hepatocellular carcinomas. Int J Cancer 105: 527-532, 2003

31. Bian EB, Zhao B, Huang C, Wang H, Meng XM, Wu BM, Ma TT, Zhang L, Lv XW and Li J: New advances of DNA methylation in liver fibrosis, with special emphasis on the crosstalk between microRNAs and DNA methylation machinery. Cell Signal 25: 1837-1844, 2013

32. Foltz G, Yoon JG, Lee H, Ryken TC, Sibenaller Z, Ehrich M, Hood L and Madan A: DNA methyltransferase-mediated transcriptional silencing in malignant glioma: A combined whole-genome microarray and promoter array analysis. Oncogene 28: 2667-2677, 2009

33. Vogelstein B, Lane D and Levine AJ: Surfing the p53 network. Nature 408: 307-310, 2000.

34. Lu KH, Li W, Liu XH, Sun M, Zhang ML, Wu WQ, Xie WP and Hou YY: Long non-coding RNA MEG3 inhibits NSCLC cells proliferation and induces apoptosis by affecting p53 expression. BMC Cancer 13: 461, 2013.

35. Zhang X, Rice K, Wang Y, Chen W, Zhong Y, Nakayama Y, Zhou Y and Klibanski A: Maternally expressed gene 3 (MEG3) noncoding ribonucleic acid: Isoform structure, expression, and functions. Endocrinology 151: 939-947, 2010. 